\title{
Weighted sums for i.i.d. random variables with relatively thin tails
}

\author{
HARTMUT LANZINGER* and ULRICH STADTMÜLLER** \\ Universität Ulm, Abt. Math. III, 89069 Ulm, Germany. \\ E-mail: *lanzinge@mathematik.uni-ulm.de; ${ }^{* *}$ stamue@mathematik.uni-ulm.de
}

We prove almost sure limit theorems for weighted sums of i.i.d. random variables with weights having a very short span, so that rather strong moment conditions are needed. This complements results due to Li et al. and the first author.

Keywords: law of the iterated logarithm; speed of convergence; strong laws of large numbers; weighted means of i.i.d. random variables

\section{Introduction}

We want to investigate the almost sure behaviour of weighted sums

$$
T_{n}=\sum_{k} a_{n k} X_{k}, \quad n \in \mathbb{N},
$$

with independently and identically distributed (i.i.d.) random variables $X, X_{1}, X_{2}, \ldots$ and a matrix of weights $A=\left(a_{n k}\right)_{n, k=1}^{\infty}$ satisfying

$$
\sum_{k} a_{n k}^{2}<\infty \quad \text { for each } n \in \mathbb{N}
$$

so that the sequence $\left(T_{n}\right)$ is well defined provided that $\mathrm{E} X^{2}<\infty$. In this case $\mathrm{E}|X|<\infty$ and we may set $\mathrm{E} X=0$. Those assumptions will be made throughout the paper without further mention. The condition (1.2) is obviously satisfied if with some sequence $\left(r_{n}\right) \in \mathbb{N}$ we have $a_{n k}=0$ for $k>r_{n}, n \in \mathbb{N}$. We are interested in random variables $X$ with relatively thin tails, i.e. we will assume that for some $p>0$,

$$
M_{p}(t):=\mathrm{Ee}^{\operatorname{tg} p(X)}:=\mathrm{E}\left(\mathrm{e}^{t|X|^{1 / p} \operatorname{sign}(X)}\right)<\infty \text { in a neighbourhood of } t=0 .
$$

There is a huge literature on strong laws for weighted sums of i.i.d. random variables; see, e.g., Chow and Lai (1973), Chow and Teicher (1978), Lai (1974a; 1974b), Bingham (1984), Bingham and Tenenbaum (1986), Bingham and Stadtmüller (1990), Bingham and Maejima (1985), Déniel and Dérriennic (1988), Heinkel (1990) and references therein. The first starting point for this paper was a recent investigation by Li et al. (1995) on weighted sums of i.i.d. random variables, mostly under moment conditions of type $\mathrm{E}\left(|X|^{p}\right)<\infty$ for some $p>1$. The second starting point were results by the first author on running means of random 
variables satisfying (1.3) for $p>1$; cf. also related results by Bingham and Tenenbaum (1986), Bingham and Goldie (1988), de Acosta and Kuelbs (1983). It was shown in Lanzinger $(1995 ; 1998)$, with $S_{n}=\sum_{k=1}^{n} X_{k}$ and $t_{1}, t_{2}>0$, that, assuming $\mathrm{E}(X)=0$,

$$
M_{p}(t)<\infty \text { for }-t_{1}<t<t_{2} \text { and } M_{p}(t)=\infty \text { for } t \in\left[-t_{1}, t_{2}\right]^{c}
$$

implies

$$
-\frac{1}{t_{1}^{p}}=\liminf _{n \rightarrow \infty} \frac{S_{n+\left[\log p^{p} n\right]}-S_{n}}{\log ^{p} n} \leqslant \limsup _{n \rightarrow \infty} \frac{S_{n+\left[\log ^{p} n\right]}-S_{n}}{\log ^{p} n}=\frac{1}{t_{2}^{p}}
$$

and that (1.5) implies (1.4) provided that $\mathrm{E}(X)=0$. We are going to show that the implication from (1.4) to (1.5) holds also for $\left(T_{n}\right)$ with a large class of weights. Hence the weights

$$
a_{n k}= \begin{cases}1 & \text { for } n+1 \leqslant k \leqslant n+1+\left[\log ^{p} n\right] \\ 0 & \text { otherwise }\end{cases}
$$

are already showing the worst but typical case, meaning that our results are in particular of interest if the weights are concentrated on a relatively short span. For a certain class of weights we shall also discuss necessity of the moment condition (1.4).

\section{Main results}

We begin with the cases $p \geqslant 1$ and sufficient conditions for almost sure convergence.

Theorem 1. Suppose in addition to our basic assumptions that with some $p \geqslant 1$ there exist $t_{1}, t_{2}>0$ such that

$$
M_{p}(t)<\infty \quad \text { for }-t_{1}<t<t_{2},
$$

and non-negative weights $a_{n k}$ satisfying

$$
\sum_{k} a_{n k}^{2}=o\left(\log ^{2 p-1} n\right)
$$

and

$$
\limsup _{n \rightarrow \infty} \sup _{k} a_{n k}=\rho<\infty
$$

Then we have

$$
-\frac{\rho}{t_{1}^{p}} \leqslant \liminf _{n \rightarrow \infty} \frac{T_{n}}{\log ^{p} n} \leqslant \limsup _{n \rightarrow \infty} \frac{T_{n}}{\log ^{p} n} \leqslant \frac{\rho}{t_{2}^{p}} \text { a.s. }
$$

Corollary 1. Under the assumptions of Theorem 1 we have

$$
\sum_{n=2}^{\infty} n^{r} P\left(T_{n}>x \log ^{p} n\right)<\infty \text { for all } x, r \geqslant 0 \text { such that } \frac{x}{(r+1)^{p}}>\frac{\rho}{t_{2}^{p}},
$$


and

$$
\sum_{n=2}^{\infty} n^{r} P\left(T_{n}<-x \log ^{p} n\right)<\infty \text { for all } x, r \geqslant 0 \text { such that } \frac{x}{(r+1)^{p}}>\frac{\rho}{t_{1}^{p}}
$$

Corollary 2. In the case $\rho=0$ or $t_{1}=t_{2}=\infty$ we have that

$$
\frac{T_{n}}{\log p^{p} n} \stackrel{\text { a.s. }}{\rightarrow} 0 \text {. }
$$

\section{Remarks 1.}

(i) The moment condition implies that for any $c \in\left(0, t_{2}\right)$ and $d \in\left(0, t_{1}\right)$ there exists some $M>0$ such that

$$
1-F_{X}(s) \leqslant \mathrm{e}^{-c s^{1 / p}} \quad \text { and } \quad F_{X}(-s) \leqslant e^{-d s^{1 / p}} \quad \text { for } s>M .
$$

(ii) If the $o(\cdot)$ in condition (2.2) is replaced by $O(\cdot)$, the proof of Theorem 1 still yields

$$
-\infty<\liminf _{n \rightarrow \infty} \frac{T_{n}}{\log ^{p} n} \leqslant \limsup _{n \rightarrow \infty} \frac{T_{n}}{\log ^{p} n}<\infty \text { a.s. }
$$

although the special form of the lim inf and lim sup given in (2.4) is not valid in general.

(iii) If we only are to prove the inequality on the right-hand side then it suffices to impose the condition that $X$ has a finite variance instead of $M_{p}(t)<\infty$ for some $t<0$ since only these conditions are used in the proof of the upper inequality (compare in particular the chain of inequalities preceeding (4.5)).

(iv) If the condition that the weights $a_{n k}$ be non-negative is dropped a similar result remains valid. With

$$
\rho=\limsup _{n \rightarrow \infty} \sup _{k}\left|a_{n k}\right|
$$

we obtain

$$
-\frac{\rho}{\min \left\{t_{1}^{p}, t_{2}^{p}\right\}} \leqslant \liminf _{n \rightarrow \infty} \frac{T_{n}}{\log ^{p} n} \leqslant \limsup _{n \rightarrow \infty} \frac{T_{n}}{\log ^{p} n} \leqslant \frac{\rho}{\min \left\{t_{1}^{p}, t_{2}^{p}\right\}} \text { a.s. }
$$

(v) The results are best possible in a certain sense, namely the denominator $\log ^{p} n$ in (2.7) can in general not be replaced by $\lambda_{n} \log ^{p} n$ with some sequence $\lambda_{n} \rightarrow 0$; see, e.g., (1.5).

Furthermore, setting

$$
a_{n k}= \begin{cases}1 & \text { if } k=n \\ 0 & \text { otherwise }\end{cases}
$$

such that $T_{n}=X_{n}$, the usual Borel-Cantelli argument shows that under the moment conditions $M_{p}(t)<\infty$ for $t \in\left(-t_{1}, t_{2}\right)$ and $M_{p}(t)=\infty$ for $t \notin\left[-t_{1}, t_{2}\right]$ we have 


$$
\limsup _{n \rightarrow \infty} \frac{T_{n}}{\log ^{p} n}=\frac{1}{t_{2}^{p}} \text { a.s. }
$$

This particularly means that the bounds given in Theorem 1 are sharp. But this is also the case for a more interesting class of weights, as can be seen from Theorem 2 below.

Similarly, consider the weights

$$
a_{n k}= \begin{cases}(-1)^{n} & \text { if } k=[n / 2] \\ 0 & \text { otherwise }\end{cases}
$$

giving rise to the sequence $T_{2 n}=X_{n}, T_{2 n+1}=-X_{n}$. As above we obtain

$$
\limsup _{n \rightarrow \infty} \frac{T_{2 n}}{(\log 2 n)^{p}}=\frac{1}{t_{2}^{p}} \text { and } \limsup _{n \rightarrow \infty} \frac{T_{2 n+1}}{(\log 2 n+1)^{p}}=\frac{1}{t_{1}^{p}} \text { a.s., }
$$

which implies the sharpness of the bounds in the version of Remark 1(iv).

Example 1. In the case of running means with an arbitrary sequence $\left(\alpha_{n}\right) \geqslant 0$ and

$$
a_{n k}= \begin{cases}1 & \text { for } \alpha_{n}+1 \leqslant k \leqslant \alpha_{n}+\left[\log ^{p} n\right], \\ 0 & \text { otherwise, }\end{cases}
$$

our assumptions are satisfied for $p>1$ with $\rho=1$ and

$$
\sum_{k} a_{n k}^{2}=\left[\log ^{p} n\right]=o\left(\log ^{2 p-1} n\right)
$$

for any such sequence $\left(\alpha_{n}\right)$.

In the case $p=1$ we only have

$$
\sum_{k} a_{n k}^{2}=\left[\log ^{p} n\right]=O\left(\log ^{2 p-1} n\right)
$$

and thus Theorem 1 yields boundedness of the running means. But in this situation much better results are known since the so-called Erdős-Rényi law applies; cf., e.g., Csörgö and Révész (1981).

Example 2. In the context of some summability methods the following weights occur (see, e.g., Stadtmüller 1995):

$$
a_{n k}=\exp \left(-\frac{1}{2}\left(\frac{k-n}{\log ^{p} n}\right)^{2}\right)
$$

For $p>1$ our conditions are obviously satisfied with $\rho=1$ and $\sum a_{n k}^{2} \asymp$ $\log ^{p} n=o\left(\log ^{2 p-1} n\right)$. The centring sequence $(n)$ can again be replaced by any non-negative sequence $\left(\alpha_{n}\right)$. For the case $p=1$ again an Erdős-Rényi law holds; see Kiesel and Stadtmüller (1996).

We now take a somewhat closer look at the special case where the weights are 
determined by some suitable sequence $\left(c_{k}\right)_{k=1}^{\infty}$ generalizing a result by Chow and Lai (1973).

Theorem 2. Apart from the basic assumptions, we assume that we are given a sequence $\left(c_{k}\right)_{k=0}^{\infty}$ of non-negative reals such that $c_{k} \rightarrow 0$ as $k \rightarrow \infty$ and

$$
\sum_{k=1}^{n} c_{k}^{2}=o\left((\log n)^{2 p-1}\right) \quad(n \rightarrow \infty)
$$

for some $p \geqslant 1$. Let $t_{1}, t_{2}>0$ and set $\rho=\max _{k \in \mathbb{N}} c_{k}$. Then the following are equivalent:

(i) (moment condition)

$$
M_{p}(t)<\infty \text { for all } t \in\left(-t_{1}, t_{2}\right)
$$

(ii) (strong law)

$$
-\frac{\rho}{t_{1}^{p}} \leqslant \liminf _{n \rightarrow \infty} \frac{1}{\log ^{p} n} \sum_{k=1}^{n} c_{n-k+1} X_{k} \text { a.s. }
$$

and

$$
\limsup _{n \rightarrow \infty} \frac{1}{\log ^{p} n} \sum_{k=1}^{n} c_{n-k+1} X_{k} \leqslant \frac{\rho}{t_{2}^{p}} \text { a.s.; }
$$

(iii) (Baum-Katz law)

$$
\sum_{n=2}^{\infty} n^{r} P\left(\sum_{k=1}^{n} c_{n-k+1} X_{k}<-x \log ^{p} n\right)<\infty
$$

for all $x, r \geqslant 0$ such that $x /(r+1)^{p}>\rho / t_{1}^{p}$ and

$$
\sum_{n=2}^{\infty} n^{r} P\left(\sum_{k=1}^{n} c_{n-k+1} X_{k}>x \log ^{p} n\right)<\infty
$$

for all $x, r \geqslant 0$ such that $x /(r+1)^{p}>\rho / t_{2}^{p}$.

\section{Remarks 2.}

(i) If the moment condition in (i) is sharp in the sense that $M_{p}(t)=\infty$ holds for $t \in\left[-t_{1}, t_{2}\right]^{c}$, we have equalities in (ii) and divergence of the series in (iii) if the inequality signs in the conditions on $x$ are reversed.

(ii) The condition that $c_{k} \rightarrow 0$ as $k \rightarrow \infty$ is only needed for equivalences involving (ii).

Next we shall discuss the case $p \in(0,1)$, i.e. the tails of $F_{X}$ are very thin. So these distributions are no longer subexponential. We shall change the notation and use $\tilde{p}=1 / p>1$ and the conjugate index $\tilde{q}$, i.e. $1 / \tilde{q}+1 / \tilde{p}=1$.

Theorem 3. Besides the basic assumptions we assume that for some $\tilde{p}>1$ there exists some $t_{0}>0$ such that 


$$
\mathrm{E}\left(\mathrm{e}^{t|X|^{\tilde{p}}}\right)<\infty \text { for }|t|<t_{0} .
$$

Suppose that for any $\kappa>0$

$$
\sum_{k:\left|a_{n k}\right| \log ^{1 / \tilde{q}} n / d_{n}<\kappa}\left(\frac{a_{n k}}{d_{n}}\right)^{j}=o\left((\log n)^{1-j / \tilde{q}}\right),
$$

uniformly in $j \geqslant 2$ with $d_{n}:=\left(\sum_{k}\left|a_{n k}\right|^{\tilde{q}}\right)^{1 / \tilde{q}}$. Then we have

$$
\limsup _{n \rightarrow \infty} \frac{\left|T_{n}\right|}{d_{n} \log ^{1 / \tilde{p}} n} \leqslant \frac{1}{t_{0}^{1 / \tilde{p}}} \text { a.s.; }
$$

in particular,

$$
\frac{T_{n}}{d_{n} \log ^{1 / \tilde{p}} n} \stackrel{\text { a.s. }}{\longrightarrow} 0 \text { in the case } t_{0}=\infty \text {. }
$$

Corollary 4. The assertion of Theorem 3 particularly holds if (2.20) is replaced by the stronger condition

$$
\min _{j: a_{n j} \neq 0}\left\{\frac{\left|a_{n j}\right|}{d_{n}} \log ^{1 / \tilde{q}} n\right\} \rightarrow \infty
$$

Example 1 (continued). If $a_{n k}=1$ for $\alpha_{n}+1 \leqslant k \leqslant \alpha_{n}+[\log p n]$ and 0 otherwise, with some sequence $\left(\alpha_{n}\right) \geqslant 0$, then the corollary applies with $d_{n} \sim \log ^{1 /(\tilde{p} \tilde{q})} n$. (Compare with de Acosta and Kuelbs (1983, Theorem 8.1) in the case $\alpha_{n}=n$; this result shows that (2.21) cannot be improved in general.)

Example 2 (continued). If

$$
a_{n k}=\exp \left(-\frac{1}{2}\left(\frac{k-n}{\log ^{1 / \tilde{p}} n}\right)^{2}\right) / \sqrt{2 \pi \log ^{2} / \tilde{p} n} \text { for } k \in \mathbb{N}_{0},
$$

then we obtain that $d_{n} \asymp \log { }^{-1 / \tilde{p}^{2}} n$. This implies that for any $\kappa>0$ (uniformly in $j$ )

$$
\sum_{k: \frac{a_{n k}}{d_{n}} \log ^{1 / \tilde{q}} n<\kappa}\left(\frac{a_{n k}}{d_{n}}\right)^{j}=O\left((\log n)^{\frac{1}{p}-\frac{j}{q}}\right)=o\left((\log n)^{1-j / \tilde{q}}\right) .
$$

Example 3. If $a_{n k}=1 /(n-k+1)$ for $0 \leqslant k \leqslant n$ and 0 otherwise, then we obtain $1 \leqslant$ $d_{n} \leqslant d_{\infty}<\infty$ and

$$
\sum_{k: a_{n k} \log ^{1 / \tilde{q}} n<\kappa} a_{n k}^{j}=\sum_{k>\log ^{1 / \tilde{q}_{\mathcal{K}^{-1}}}} \frac{1}{k^{j}} \asymp(\log n)^{(1-j) /(\tilde{q})}=o\left((\log n)^{1-j / \tilde{q})}\right.
$$

uniformly in $j$. So condition (2.20) is satisfied for all $\tilde{p}>1$. 
Example 4. If $a_{n k}=c_{n-k}$ with some sequence $\left(c_{k}\right)_{0}^{\infty} \in \ell_{2}$, then condition (2.20) reads

$$
\sum_{k: c_{k} \log 1 / \tilde{q}} c_{n<\kappa}^{j}=o\left((\log n)^{1-j / \tilde{q}}\right) \text { uniformly in } j
$$

which is, for example, satisfied if $c_{k}=O\left(k^{-\beta}\right)$ and $\beta>1 / \tilde{q}=(\tilde{p}-1) / \tilde{p}$; this is somewhat weaker than the related condition $\beta=\tilde{p} /(\tilde{p}+1)$ in Chow and Lai (1973, Theorem 3), where the special case $t_{0}=\infty$ is discussed.

\section{Discussion of special weighted sums}

We shall discuss various strong laws based on summability methods and their speed of convergence under our moment conditions

$$
M_{p}(t)<\infty \text { in a neighbourhood of } t=0 \text { and } \mathrm{E}(X)=0
$$

with some given $p \geqslant 1$. We shall assume this moment condition everywhere in this section up to Theorem 4 . The index $k$ will now run through $\mathbb{N}_{0}$ to meet standard notation.

(i) Cesàro methods $C_{\alpha}$. Here we obtain, with $A_{\mu}^{\beta}:=\left(\begin{array}{c}\mu+\beta \\ \mu\end{array}\right)$ for $\mu \in \mathbb{N}_{0}$ and $\beta \in \mathbb{R}$,

$$
\sum_{k=0}^{n} \frac{A_{n-k}^{\alpha-1}}{A_{n}^{\alpha}} X_{k} \stackrel{\text { a.s. }}{=} \begin{cases}O\left(n^{-1 / 2}(\log n)^{1 / 2}\right) & \text { if } \alpha>\frac{1}{2} \\ O\left(n^{-\alpha}(\log n)^{p}\right) & \text { if } 0<\alpha \leqslant \frac{1}{2} .\end{cases}
$$

This follows from Remark 1(iii), formula (2.9), using

$$
a_{n k}= \begin{cases}\frac{A_{n-k}^{\alpha-1}}{A_{n}^{\alpha}} \sqrt{n}(\log n)^{p-1 / 2}, & \text { if } \alpha>\frac{1}{2}, \\ \frac{A_{n-k}^{\alpha-1}}{A_{n}^{\alpha}} n^{\alpha}, & \text { if } 0<\alpha \leqslant \frac{1}{2} .\end{cases}
$$

Observe that then $\rho=0$ for $\alpha>1 / 2$ and $\rho<\infty$ if $0<\alpha \leqslant 1 / 2$ and $\sum_{k} a_{n k}^{2}=O\left(\log ^{2 p-1} n\right)$ in the case $\alpha>1 / 2$ or $\alpha=1 / 2$ and $p=1$ and $\sum_{k} a_{n k}^{2}=o\left(\log ^{2 p-1} n\right)$ in the other cases. If $\alpha<1 / 2, \quad p=1$ and $M_{p}(t)<\infty$ for all $t$, Li et al. (1995) have shown that $O($.$) can be$ replaced by $o($.$) in the bounds above.$

For $\alpha \geqslant 1$ these rates are not optimal, since there exists a law of iterated logarithm (LIL) yielding the rate $O\left(n^{-1 / 2} \sqrt{\log \log n}\right)$. For $\alpha \in(1 / 2,1)$, Li et al. (1995) conjectured that a LIL is valid as well. This will be proven (with a somewhat different constant than conjectured) next.

Theorem 4. For $1 / 2<\alpha<1$ the following statements are equivalent:

(i) $\mathrm{E}(X)=0, \mathrm{E}\left(X^{2}\right)=1$ and

$$
\text { (ii) } \limsup _{n \rightarrow \infty} \sqrt{\frac{\mathrm{E}\left(|X|^{2 /(2 \alpha-1)} /\left(\log \log \left(\mathrm{e}^{\mathrm{e}}+|X|\right)\right)^{1 /(2 \alpha-1)}\right)<\infty}{2 \alpha^{2}(2 \alpha-1)^{-1} \log \log n}} \sum_{k=0}^{n} \frac{A_{n-k}^{\alpha-1}}{A_{n}^{\alpha}} X_{k} \stackrel{\text { a.s. }}{=} 1 .
$$


Remark 3. In the case $\alpha=1$ we have the classical law of iterated logarithm, where, however, precisely the second moment is needed. For bounded random variables the cases $\alpha \geqslant 1$ have already been discussed by Gaposhkin (1965); for more general random variables, see Lai (1974b). The problem remains of what happens in the cases $\alpha \leqslant 1 / 2$.

Proof. The implication (i) $\Rightarrow$ (ii) follows from a slightly more general version of Stadtmüller (1984, Theorem 1 and Corollary 1). Under our moment condition we have the following approximation quality in strong approximation of $S_{n}$ by a Wiener process $W(n)$ (see, for example, Csörgő and Révész 1981, Section 2.6):

$$
S_{n}-W(n) \stackrel{\text { a.s. }}{=} o\left(n^{\alpha-1 / 2}(\log \log n)^{1 / 2}\right) .
$$

Putting $a_{n k}=n^{1 / 2+\epsilon} A_{n-k}^{\alpha-1} / A_{n}^{\alpha}$ and $s_{n}^{2}=\sum_{k=0}^{n} a_{n k}^{2} \sim n^{2 \epsilon} \frac{\alpha^{2}}{2 \alpha-1} \nearrow \infty$, condition (1.5b) in Stadtmüller (1984) reads as follows

$$
n^{\alpha-1 / 2} \sqrt{\log \log n}\left(\sum_{k=1}^{n}\left|a_{n k}-a_{n, k-1}\right|+\left|a_{n n}\right|\right) / \sqrt{n^{2 \epsilon} \log \log n}=O(1)
$$

which is satisfied since $\sum_{k=1}^{n}\left|a_{n k}-a_{n, k-1}\right|+\left|a_{n n}\right|=O\left(n^{1 / 2+\epsilon-\alpha}\right)$. The other conditions required in Stadtmüller (1984) can be satisfied easily since $s_{n}^{2}$ is a smooth function in $n$ and since $\left(\sum_{k=0}^{m} a_{n k} a_{m k}\right) /\left(\sum_{k=0}^{n} a_{n k}^{2}\right)$ is smooth in $m / n$.

The implication (ii) $\Rightarrow_{p}$ (i) follows by standard arguments using the original weights. Since $X_{n} /\left(n^{\alpha-1 / 2} \sqrt{\log \log n}\right) \stackrel{p}{\rightarrow} 0$ we obtain by Lemma 1 in Lai $(1974 b)$, with the variables

$$
Z_{n}=X_{n} /\left(n^{\alpha-1 / 2} \sqrt{\log \log n}\right) \quad \text { and } \quad Y_{n}=\sum_{k=0}^{n-1} \frac{A_{n-k}^{\alpha-1}}{A_{n}^{\alpha}} X_{k} / \sqrt{n^{-1 / 2} \log \log n},
$$

that

$$
X_{n} /\left(n^{\alpha-1 / 2} \sqrt{\log \log n}\right) \stackrel{\text { a.s. }}{=} O(1)
$$

Now we use the fact that the sequence $\left(X_{k}\right)$ is i.i.d and apply the Borel-Cantelli lemma to obtain, for some $x$ large enough,

$$
\sum_{n=1}^{\infty} P\left(\left|X_{1}\right|>x n^{\alpha-1 / 2}(\log \log n)^{1 / 2}\right)<\infty
$$

which in turn gives the desired moment condition. We then must have $\mathrm{E} X=0$ and $\mathrm{E} X^{2}=\sigma^{2}$.

(ii) Euler methods $E_{p}$. Here we have

$$
\sum_{k=0}^{n}\left(\begin{array}{l}
n \\
k
\end{array}\right) p^{k}(1-p)^{n-k} X_{k} \stackrel{\text { a.s. }}{=} O\left(n^{-1 / 4} \sqrt{\log n}\right) \text {. }
$$

Again Remark 1(iii), formula (2.9), applies with $a_{n k}=\left(\begin{array}{c}n \\ k\end{array}\right) p^{k}(1-p)^{n-k} n^{1 / 4}(\log n)^{p-1 / 2}$. Then we obtain $\rho=0$ and $\sum_{k} a_{n k}^{2}=O\left(\log ^{2 p-1} n\right)$. The rate in (3.2) is optimal since there is 
a law of the single logarithm - see, for example, Lai (1974b) and a discussion of the correct constant in Bingham (1984) - saying

$$
\limsup _{n \rightarrow \infty} \sqrt{\frac{2 \sqrt{\pi n}}{\log n}} \sum_{k=0}^{n}\left(\begin{array}{l}
n \\
k
\end{array}\right) p^{k}(1-p)^{n-k} X_{k} \stackrel{\text { a.s. }}{=} \sqrt{\operatorname{var}\left(X_{1}\right) \sqrt{(p-1) / p}},
$$

which, however, requires a more detailed analysis.

(iii) Random walk methods. If we define $p_{n k}=P\left(T_{n}=k\right)$, where $T_{n}=\sum_{j=1}^{n} Y_{j}$ with i.i.d. non-periodic random variables $\left(Y_{j}\right), Y: \Omega \rightarrow \mathbb{N}_{0}$, having finite third moments, a maximal span of 1 and satisfying $\mathrm{E}(Y)=\mu$ and $\mathrm{E}\left(Y^{2}\right)=\sigma^{2}$, then we have

$$
\sum_{k=0}^{\infty} p_{n k} X_{k} \stackrel{\text { a.s. }}{=} O\left(n^{-1 / 4} \sqrt{\log n}\right),
$$

and in particular this gives for the Borel method $\sum_{k=0}^{\infty} \mathrm{e}^{-n \frac{n^{k}}{k !}} X_{k} \stackrel{\text { a.s. }}{=} O\left(n^{-1 / 4} \sqrt{\log n}\right)$. All these rates are optimal since there is again a law of the single logarithm - see, for example, Bingham (1984) and Lai (1974a). Our result above follows from the following facts:

(a) $X_{n} \stackrel{\text { a.s }}{=} O\left(\log ^{p} n\right)$;

(b) a local central limit theorem (see, for example, Petrov 1975) holds, according to which

$$
p_{n k}=\frac{1}{\sqrt{2 \pi n \sigma^{2}}} \exp \left(-\frac{1}{2}\left(\frac{k-n \mu}{\sqrt{n \sigma^{2}}}\right)^{2}\right)\left(1+\frac{\gamma_{3}}{6 \sigma^{3} \sqrt{n}} H_{3}\left(\frac{k-n \mu}{\sqrt{n \sigma^{2}}}\right)\right)+o\left(\frac{n^{-1}}{1+\left|\frac{k-n \mu}{\sqrt{n \sigma^{2}}}\right|^{3}}\right)
$$

uniformly in $k$ with $\gamma_{3}$ being the third cumulant of $Y$ and $H_{3}$ the third Hermite polynomial, yielding $T_{n}=V_{\sqrt{n}}+o\left(n^{-1 / 2} \log ^{p} n\right)$, where $V_{\sqrt{n}}$ is defined below;

(c) the results on Valiron $\left(V_{\sqrt{n}}\right)$ means discussed next.

(iv) Valiron means $V_{\phi_{n}}$. Here we use a positive sequence $\left(\phi_{n}\right)$ satisfying $\phi_{n} / \log ^{p} n \rightarrow \infty$ and we shall show that

$$
\begin{aligned}
V_{\phi(n)} & =\sum_{k=0}^{\infty} \frac{1}{\sqrt{2 \pi \phi_{n}^{2}}} \exp \left(-\frac{1}{2}\left(\frac{k-n}{\phi_{n}}\right)^{2}\right) X_{k} \\
& \stackrel{\text { a.s. }}{=} \begin{cases}O\left(\phi_{n}^{-1 / 2} \sqrt{\log n}\right), & \text { if } \phi_{n} / \log ^{2 p-1} n \geqslant \delta>0, \\
O\left((\log n)^{p} / \phi_{n}\right), & \text { if } \phi_{n} / \log ^{2 p-1} n \rightarrow 0 .\end{cases}
\end{aligned}
$$

Therefore we have to consider

$$
a_{n k}=\frac{1}{\sqrt{2 \pi} \phi_{n}} \exp \left(-\frac{1}{2}\left(\frac{k-n}{\phi_{n}}\right)^{2}\right) b_{n}
$$

where 


$$
b_{n}= \begin{cases}\phi_{n}^{1 / 2} \log p-1 / 2 & \text { if } \phi_{n} / \log ^{2 p-1} n \geqslant \delta>0, \\ \phi_{n} & \text { otherwise. }\end{cases}
$$

Special cases are $\phi_{n}=\sqrt{n}$ and $\phi_{n}=\log ^{p} n$; however, the second case was discussed in Section 2 and does not satisfy the basic assumption on $\phi_{n}$ here.

(v) Running means. Here we are going to verify that for the same sequences $\left(\phi_{n}\right)$ as in (iv) we have, for any $u>0$,

$$
\frac{1}{u \phi_{n}} \sum_{n<k \leqslant n+u \phi_{n}} X_{k}= \begin{cases}O\left(\phi_{n}^{-1 / 2} \sqrt{\log n}\right) & \text { if } \phi_{n} / \log ^{2 p-1} n \geqslant \delta>0 \\ O\left((\log n)^{p} / \phi_{n}\right) & \text { if } \phi_{n} / \log ^{2 p-1} n \rightarrow 0\end{cases}
$$

This follows from the choice

$$
a_{n k}=\left\{\begin{array}{lll}
\phi_{n}^{-1 / 2} \log ^{p-1 / 2} n, & \text { for } n<k \leqslant n+u \phi_{n}, & \text { if } \phi_{n} / \log ^{2 p-1} n \geqslant \delta>0 \\
1, & \text { for } n<k \leqslant n+u \phi_{n}, & \text { otherwise. }
\end{array}\right.
$$

The case $\phi_{n} \sim c \log ^{p} n$ was discussed in Section 2. The rates are optimal again in the case $\phi_{n}=\sqrt{n}$ (see, for example, Lai 1974a). A discussion of relations between (iii) and (v) can be found in Stadtmüller (1995). We remark that the result on running means contains situations not yet discussed in the literature, such as $p=2, \phi_{n}=\log ^{p+1 / 2} n$. For both cases, the Valiron and the running means, the centring at $(n)$ can be replaced by a non-negative sequence $\left(\alpha_{n}\right)$.

\section{Proofs}

Proof of Theorem 1. It suffices to prove the inequality for the upper limit. Let $\epsilon \in\left(0, \mathrm{t}_{2}\right)$ and $s_{2}=t_{2}-\epsilon$. Consider the decomposition $X_{k}=X_{n k}^{\prime}+X_{n k}^{\prime \prime}$ with

$$
X_{n k}^{\prime}=X_{k} \cdot 1_{\left\{X_{k} \leqslant s_{2}^{-p}((1+\epsilon) \log n)^{p}\right\}}
$$

and $X_{n k}^{\prime \prime}=X_{k}-X_{n k}^{\prime}$. Note that $\mathrm{E} X_{n k}^{\prime}+\mathrm{E} X_{n k}^{\prime \prime}=0$ and

$$
\frac{T_{n}}{\log ^{p} n}=\sum_{k=1}^{\infty} \frac{a_{n k}}{\log ^{p} n}\left(X_{n k}^{\prime}-\mathrm{E} X_{n k}^{\prime}\right)+\sum_{k=1}^{\infty} \frac{a_{n k}}{\log ^{p} n}\left(X_{n k}^{\prime \prime}-\mathrm{E} X_{n k}^{\prime \prime}\right)=I_{n}+\mathrm{II}_{n} .
$$

Markov's inequality implies

$$
\begin{aligned}
& P\left(\mathrm{II}_{n}>\epsilon\right) \leqslant \frac{1}{\epsilon^{2} \log ^{2} p} \sum_{k=1}^{\infty} a_{n k}^{2} \mathrm{E} X_{n k}^{\prime \prime 2} \\
& =\frac{1}{\epsilon^{2} \log ^{2 p} n} \sum_{k=1}^{\infty} a_{n k}^{2} \int\left\{t>s_{2}^{-p}((1+\epsilon) \log n)^{p}\right\} t^{2} \mathrm{e}^{-s_{2} t^{1 / p}} \mathrm{e}^{s_{2} t^{1 / p}} \mathrm{~d} F_{x}(t) \\
& \leqslant \frac{1}{\epsilon^{2} \log ^{2 p} n} \sum_{k=1}^{\infty} a_{n k}^{2} \mathrm{e}^{-(1+\epsilon) \log n} \mathrm{E}\left(X^{2} \mathrm{e}^{s_{2} g_{p}(X)}\right),
\end{aligned}
$$

and hence $\sum_{n=1}^{\infty} P\left(\mathrm{II}_{n}>\epsilon\right)<\infty$. Thus we obtain 


$$
\limsup _{n \rightarrow \infty} \mathrm{II}_{n} \leqslant \epsilon \text { a.s. }
$$

by the Borel-Cantelli lemma.

Note that $\mu_{n}=\mathrm{E} X_{n k}^{\prime} \leqslant 0$ and

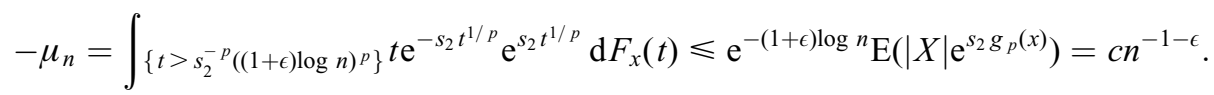

Turning to $I_{n}$, we set $t=s_{2}^{p} /\left((\rho+\epsilon)(1+\epsilon)^{p-1}\right)$ and $x=(1+\epsilon) / t$. Noting that $x \leqslant$ $\rho / t_{2}^{p}+\kappa \epsilon$, with $\kappa$ independent of $\epsilon$, we obtain

$$
P\left(I_{n}>x\right) \leqslant n^{-t x} \prod_{k=1}^{\infty} \mathrm{E}\left(\exp \left\{t \frac{a_{n k}}{\log p-1}\left(X_{n k}^{\prime}-\mu_{n}\right)\right\}\right) .
$$

Now we have

$$
\begin{aligned}
\mathrm{E}\left(\exp \left\{\frac{a_{n k} t}{\log p^{p-1} n}\left(X_{n k}^{\prime}-\mu_{n}\right)\right\}\right) & =\exp \left\{\frac{-a_{n k} t}{\log p^{p-1} n} \mu_{n}\right\} \mathrm{E}\left(\exp \left\{\frac{a_{n k} t}{\log p-1} X_{n k}^{\prime}\right\}\right) \\
& \leqslant \exp \left\{\frac{\rho+\epsilon}{\log p-1} \frac{c t}{n^{1+\epsilon}}\right\} \mathrm{E}\left(\exp \left\{\frac{a_{n k} t}{\log p-1} X_{n k}^{\prime}\right\}\right) \\
& =\mathrm{e}^{o(1)} \mathrm{E}\left(\exp \left\{t \frac{a_{n k}}{\log p-1} X_{n k}^{\prime}\right\}\right) .
\end{aligned}
$$

Choose $q$ such that $p^{-1}+q^{-1}=1$ and set $H(x)=\max \left\{1, \mathrm{e}^{x}\right\}$. By $\mu_{n} \leqslant 0$, using successively (2.3), (2.2), (2.1) and the obvious inequality $\mathrm{e}^{x} \leqslant 1+x+\left(x^{2} / 2\right) H(x)$, we also have

$$
\begin{aligned}
& \mathrm{E}\left(\exp \left\{t \frac{a_{n k}}{\log p-1} X_{n k}^{\prime}\right\}\right) \\
& \leqslant 1+\frac{t^{2} a_{n k}^{2}}{2(\log n)^{2 p-2}} \mathrm{E}\left(\left(X_{n k}^{\prime}\right)^{2} H\left(t \frac{a_{n k}}{\log p-1} X_{n k}^{\prime}\right)\right) \\
& \leqslant 1+\frac{t^{2} a_{n k}^{2}}{2(\log n)^{2 p-2}} \cdot\left\{\mathrm{E} X^{2}+\mathrm{E}\left(X^{2} \cdot \mathscr{X}_{\{X \geqslant 0\}} \exp \left\{t \frac{a_{n k}}{\log p^{p-1} n}\left(\frac{(1+\epsilon) \log n}{s_{2}}\right)^{p / q}|X|^{1 / p}\right\}\right)\right\} \\
& \leqslant 1+\frac{t^{2} a_{n k}^{2}}{2(\log n)^{2 p-2}} O(1) \leqslant \exp \left\{\frac{t^{2} a_{n k}^{2}}{2(\log n)^{2 p-2}} O(1)\right\} .
\end{aligned}
$$

Together with (4.4), we have

$$
P\left(I_{n}>x\right) \leqslant n^{-t x} \exp \left\{\sum_{k=1}^{\infty} \frac{t^{2} a_{n k}^{2}}{2(\log n)^{2 p-2}} O(1)\right\}=n^{-t x} \mathrm{e}^{o(\log n)}
$$

which yields a convergent series since $t x>1$. Thus we have proved 


$$
\limsup _{n \rightarrow \infty} I_{n} \leqslant x \leqslant \frac{\rho}{t_{2}^{p}}+\kappa \epsilon \text { a.s. }
$$

with some $\kappa>0$. Therefore

$$
\limsup _{n \rightarrow \infty} \frac{T_{n}}{\log ^{p} n} \leqslant \frac{\rho}{t_{2}^{p}}+\kappa \epsilon+\epsilon \text { a.s. }
$$

Since the choice of $\epsilon \in\left(0, t_{2}\right)$ was arbitrary, this proves the assertion.

Proof of Corollary 1. This only requires a look at the proof above. Let $x /(r+1)^{p}>\rho / t_{2}^{p}$. Choose $s_{2} \in\left(0, t_{2}\right), c>r+1$ and $\delta>0$ such that

$$
\frac{x-\delta}{c^{p}}>\frac{\rho+\delta}{s_{2}^{p}} .
$$

Replace $(1+\epsilon) \log n$ by $c \log n$ in the truncation used in the proof above, set $t=c /(x-\delta)$ and observe that for all large $n$ and all $k$

$$
\frac{t a_{n k}(c \log n)^{p-1}}{(\log n)^{p-1} s_{2}^{p-1}} \leqslant \frac{c}{x-\delta} \cdot \frac{c^{p-1}(\rho+\delta)}{s_{2}^{p-1}}<s_{2} .
$$

Now the proof of Theorem 1 implies

$$
\begin{aligned}
& P\left(T_{n}>x \log ^{p} n\right) \\
& \quad \leqslant P\left(\sum_{k=1}^{\infty} a_{n k}\left(X_{n k}^{\prime}-\mu_{n}\right)>(x-\delta) \log ^{p} n\right)+P\left(\sum_{k=1}^{\infty} a_{n k}\left(X_{n k}^{\prime \prime}+\mu_{n}\right)>\delta \log ^{p} n\right) \\
& \quad=O\left(n^{-t(x-\delta)}\right)+O\left(n^{-c}\right)=O\left(n^{-c}\right)
\end{aligned}
$$

which yields the desired conclusion.

Proof of Remark 1(iii). Checking (4.5) again, we find that the result holds for $x$ large enough.

Proof of Theorem 2. That (i) implies (ii) as well as (iii) is an immediate consequence of Theorem 1 and Corollary 1. On the other hand, it is obvious that (iii) implies (ii) by the Borel-Cantelli lemma. So only for (ii) $\Rightarrow$ (i) is there anything left to prove. Thus we assume (ii). Let $k_{0}$ be an index with $c_{k_{0}}=\rho$. If we decompose $(\log n)^{-p} T_{n}$ into

$$
Y_{n}=\sum_{k=1}^{n-k_{0}+1} \frac{c_{n-k+1}}{\log ^{p} n} X_{k}
$$

and

$$
Z_{n}=\sum_{k=n-k_{0}+2}^{n} \frac{c_{n-k+1}}{\log ^{p} n} X_{k},
$$


then Lai (1974b, Lemma 1) shows that

$$
-\frac{\rho}{t_{1}^{p}} \leqslant \liminf _{n \rightarrow \infty} Y_{n} \leqslant \limsup _{n \rightarrow \infty} Y_{n} \leqslant \frac{\rho}{t_{2}^{p}} \text { a.s. }
$$

Now continue by decomposing $Y_{n}$ further into the summands

$$
U_{n}=\sum_{k=1}^{n-k_{0}} \frac{c_{n-k+1}}{\log ^{p} n} X_{k}
$$

and

$$
V_{n}=\frac{c_{k_{0}}}{\log { }^{p} n} X_{n-k_{0}+1}=\frac{\rho}{\log ^{p} n} X_{n-k_{0}+1} .
$$

Since $\mathrm{E} X^{2}<\infty$ and $\mathrm{E} X=0$, it is easy to see that $U_{n} \stackrel{p}{\rightarrow} 0$ as $n \rightarrow \infty$, for example, by Markov's inequality.

Fixing $\epsilon>0$, an application of a result by Martikainen (1982, Lemma 3) to the events $A_{n}=\left\{V_{n}>\rho / t_{2}^{p}+2 \epsilon\right\}$ and $B_{n}=\left\{U_{n}>-\epsilon\right\}$ shows that

$$
P\left(Y_{n}>\frac{\rho}{t_{2}^{p}}+\epsilon \text { i.o. }\right) \geqslant P\left(V_{n}>\frac{\rho}{t_{2}^{p}}+2 \epsilon \text { i.o. }\right)
$$

By independence and Borel-Cantelli this means that

$$
\sum_{n=1}^{\infty} P\left(X>\left(\frac{1}{t_{2}^{p}}+\frac{2 \epsilon}{\rho}\right) \log ^{p} n\right)<\infty .
$$

Thus recalling that $g_{p}(y)=\operatorname{sign}(y)|y|^{1 / p}$ we have

$$
\mathrm{E}\left(\exp \left\{\operatorname{tg}_{p}(X)\right\}\right)<\infty
$$

for all $t \in\left(0,\left(1 / t_{2}^{p}+2 \epsilon / \rho\right)^{-1 / p}\right)$. Since $\epsilon>0$ was arbitrary, (4.13) holds for all $t \in\left(0, t_{2}\right)$. A corresponding result for negative $t$ can be proved similarly. This yields the conclusion.

Proof of Theorem 3. We start by proving Corollary 4. Without loss of generality we may assume that $d_{n}=1$ (we only have to convince ourselves that all conditions hold for the modified weights $\tilde{a}_{n k}=a_{n k} / d_{n}$ ) and $a_{n k}=0$ for $k \geqslant r_{n}$ with some $r_{n} \in \mathbb{N}$, because if $a_{n k}$ has infinitely many non-vanishing terms then we can cut the series into two pieces

$$
T_{n}=\sum_{k=1}^{r_{n}} a_{n k} X_{k}+\sum_{k=r_{n}+1}^{\infty} a_{n k} X_{k}=T_{n}^{(1)}+T_{n}^{(2)}
$$

with arbitrarily large $r_{n}$ so that $T_{n}^{(2)} /(\log n)^{1 / \tilde{p}} \rightarrow 0$ a.s. as $n \rightarrow \infty$.

By independence and Markov's inequality we find that, for $\lambda>0$,

$$
P\left(T_{n}>x(\log n)^{1 / \tilde{p}}\right) \leqslant \exp \left\{-x \lambda(\log n)^{1 / \tilde{p}}\right\} \prod_{k=1}^{r_{n}} \mathrm{E}\left(\mathrm{e}^{\lambda\left|\mathrm{a}_{n k} \| X_{k}\right|}\right) .
$$

We now borrow an idea from de Acosta and Kuelbs (1983, Lemma 8.1) and use the 
arithmetic-geometric mean inequality. Then we obtain, with $\tilde{t_{0}}>0$ and $\alpha=(1 / \tilde{q})\left(1 / \tilde{p} \tilde{t_{0}}\right)^{\tilde{q} / \tilde{p}}$, that for $y, \lambda \geqslant 0$,

$$
y \lambda \leqslant \tilde{t_{0}} y^{\tilde{p}}+\alpha \lambda^{\tilde{q}}
$$

This yields, for $\tilde{t_{0}}<t_{0}$,

$$
\mathrm{E}\left(\mathrm{e}^{\lambda\left|a_{n k}\right| X \mid}\right) \leqslant \mathrm{E}\left(\mathrm{e}^{\tilde{t_{0}}|X| \tilde{p}}\right) \mathrm{e}^{\alpha \lambda^{\tilde{q}}\left|a_{n k}\right|^{\tilde{q}}} .
$$

For any $\epsilon>0$ there exists some $\lambda_{0}(\epsilon)$ such that, for all $\lambda \geqslant \lambda_{0}(\epsilon) /\left|a_{n k}\right|$,

$$
\mathrm{E}\left(\mathrm{e}^{\lambda\left|\mathrm{a}_{n k} \| X\right|}\right) \leqslant \mathrm{e}^{(\alpha+\epsilon) \lambda^{\tilde{q}}\left|a_{n k}\right|^{\tilde{q}}} .
$$

The last inequality is obviously true for any $\lambda$ if $a_{n k}=0$. Hence we find that for sufficiently large $\lambda$ :

$$
\prod_{k=1}^{r_{n}} \mathrm{E}\left(\mathrm{e}^{\lambda\left|\mathrm{a}_{n k} \| X\right|}\right) \leqslant \exp \left\{(\alpha+\epsilon) \lambda^{\tilde{q}} \sum_{k=1}^{r_{n}}\left|a_{n k}\right|^{\tilde{q}}\right\}=\mathrm{e}^{(\alpha+\epsilon) \lambda^{\tilde{q}}},
$$

since $d_{n}=1$. The inequality (4.15) can be minimized putting

$$
\lambda=\left(\frac{x}{\alpha^{*} \tilde{q}}\right)^{1 /(\tilde{q}-1)}(\log n)^{1 / \tilde{q}}
$$

with $\alpha^{*}=\alpha+\epsilon$. Under our assumption (2.23) $\lambda\left|a_{n k}\right| \geqslant \lambda_{0}(\epsilon)$ eventually and our inequalities above apply. Hence we obtain

$$
\begin{aligned}
P\left(T_{n}>x(\log n)^{1 / \tilde{p}}\right) & \leqslant \exp \left\{-\log n\left(x^{\tilde{q} /(\tilde{q}-1)}\left(\alpha^{*} \tilde{q}\right)^{-1 /(\tilde{q}-1)}\left(1-\tilde{q}^{-1}\right)\right)\right\} \\
& =\exp \left\{-\log n x^{\tilde{p}}\left(\left(\alpha^{*} \tilde{q}\right)^{-1 /(\tilde{q}-1)} \frac{1}{\tilde{p}}\right)\right\} .
\end{aligned}
$$

If

$$
x^{\tilde{p}}\left(\frac{1}{\alpha^{*} \tilde{q}}\right)^{1 /(\tilde{q}-1)} \frac{1}{\tilde{p}}>1,
$$

that is

$$
x>\tilde{p}^{1 / \tilde{p}}\left(\left(\frac{1}{\tilde{p} \tilde{t}_{0}}\right)^{\tilde{q} / \tilde{p}}+\epsilon \tilde{q}\right)^{1 / \tilde{q}}=\frac{1}{\tilde{t}_{0}^{1 / \tilde{p}}}+\tilde{\epsilon},
$$

we have for any $x>\frac{1}{\tilde{t}_{0}^{1 / \tilde{p}}}$ that

$$
\sum_{n=1}^{\infty} P\left(T_{n}>x(\log n)^{1 / \tilde{p}}\right)<\infty
$$

which implies that

$$
\limsup _{n \rightarrow \infty} \frac{T_{n}}{(\log n)^{1 / \tilde{p}}} \leqslant \frac{1}{t_{0}^{1 / \tilde{p}}} \text { a.s. }
$$


Turning to the proof of Theorem 3 itself, let us split $T_{n}=T_{n, 1}+T_{n, 2}$ where

$$
T_{n, j}=\sum_{k \in I_{n, j}} a_{n k} X_{k}, j=1,2,
$$

with $I_{n, 1}$ containing all indices with $\left|a_{n k}\right|>\kappa / \log { }^{1 / \tilde{q}} n$ with some constant $\kappa$ to be specified below. We begin to estimate $P\left(T_{n, 1}>(x-\epsilon) \log { }^{1 / \tilde{p}} n\right)$. Now we proceed with this part as in (a) using

$$
\kappa=\kappa(\epsilon)=\max \left\{\lambda_{0}(\epsilon)\left(\frac{\alpha^{*} \tilde{q}}{x}\right)^{1 /(\tilde{q}(\tilde{q}-1))}, 1\right\} .
$$

Observe that in (4.19) we only need $\sum_{k \in I_{n, 1}}\left|a_{n k}\right|^{\tilde{q}} \leqslant 1$. Hence

$$
\limsup _{n \rightarrow \infty} \frac{T_{n, 1}}{(\log n)^{1 / \tilde{p}}} \leqslant \frac{1}{t_{0}^{1 / \tilde{p}}}+\epsilon \text { a.s., }
$$

for all $\epsilon>0$. For the second part observe that

$$
P\left(T_{n, 2}>\epsilon \log 1 / \tilde{p} n\right) \leqslant \mathrm{e}^{-\epsilon \lambda \log 1 / \tilde{p} n} \prod_{k \in I_{n, 2}} \mathrm{E}\left(\mathrm{e}^{\lambda a_{n k} X_{k}}\right) .
$$

If $\sum_{j=2}^{\infty}\left(c_{j} / j !\right) \lambda^{j}, \lambda \in \mathbb{R}$, is the cumulant generating function of $X$ (observe that $\mathrm{E} X=0$, thus $\left.c_{1}=0\right)$ we have for the cumulant generating function of $a_{n k} X_{k}$ :

$$
\varphi_{n, k}(\lambda)=\sum_{j=2}^{\infty} \frac{c_{j} a_{n k}^{j}}{j !} \lambda^{j}
$$

and therefore

$$
\prod_{k \in I_{n, 2}} \mathrm{E}\left(\mathrm{e}^{\lambda a_{n k} X_{k}}\right)=\exp \left\{\sum_{j=2}^{\infty} \frac{c_{j}}{j !} \lambda^{j} \sum_{k \in I_{n, 2}} a_{n k}^{j}\right\},
$$

and by (2.20) we find that for any $\kappa>0$ there exists $n_{0} \in \mathbb{N}$ such that, for $n \geqslant n_{0}$,

$$
\prod_{k \in I_{n, 2}} \mathrm{E}\left(\mathrm{e}^{\lambda a_{n k} X_{k}}\right) \leqslant \exp \left\{\mathrm{o}(1) \cdot \sum_{j=2}^{\infty} \frac{\left|c_{j}\right|}{j !}(\log n)^{1-j / \tilde{q}} \lambda^{j}\right\} .
$$

Since $(\log n)^{j / \tilde{q}} \cdot(\log n)^{1-j / \tilde{q}}=\log n$ for all $n$ and $j \geqslant 2$ we find that with $\lambda_{n}=2 / \epsilon \cdot(\log n)^{1 / \tilde{q}}$,

$$
\prod_{k \in I_{n, 2}} \mathrm{E}\left(\mathrm{e}^{\lambda_{n} a_{n k} X_{k}}\right) \leqslant \exp \left\{\mathrm{o}(\log n) \cdot \sum_{j=2}^{\infty} \frac{\left|c_{j}\right|}{j !}(2 / \epsilon)^{j}\right\} \leqslant \mathrm{e}^{o(\log n)} .
$$

Hence we obtain, with $\lambda_{n}=2 / \epsilon \cdot \log { }^{1 / \tilde{q}} n$ in (4.26), that

$$
P\left(T_{n, 2}>\epsilon \log 1 / \tilde{p} n\right) \leqslant \kappa_{\epsilon} n^{-2+\epsilon},
$$

yielding that 


$$
\limsup _{n \rightarrow \infty} \frac{T_{n, 2}}{(\log n)^{1 / \tilde{p}}} \leqslant \epsilon \text { a.s. }
$$

Since $\epsilon>0$ was arbitrary, we obtain the desired result.

\section{Acknowledgements}

The authors wish to thank the unknown referees for useful hints and comments, in particular for the Martikainen (1982) reference which led to a simpler proof of Theorem 2.

\section{References}

Bingham, N.H. (1984) On Valiron and circle convergence. Math. Z., 186, 273-286.

Bingham, N.H. and Goldie, C.M. (1988) Riesz means and self-neglecting functions. Math. Z., 199, $443-454$.

Bingham, N.H. and Maejima, M. (1985) Summability methods and almost sure convergence. $Z$. Wahrscheinlichkeitstheorie Verw. Geb., 68, 383-392.

Bingham, N.H. and Stadtmüller, U. (1990) Jakimovski methods and almost sure convergence. In G.R. Grimmett and D. Welsh (eds), Disorder in Physical Systems. Oxford: Clarendon Press.

Bingham, N.H. and Tenenbaum, G. (1986) Riesz and Valiron means and fractional moments. Math. Proc. Cambridge Philos. Soc., 99, 143-149.

Chow, Y.S. and Lai, T.L. (1973) Limiting behaviour of weighted sums of independent random variables. Ann. Probab., 1, 810-824.

Chow, Y.S. and Teicher, H. (1978) Probability Theory: Independence, Interchangeability, Martingales. New York: Springer-Verlag.

Csörgö, M. and Révész, P. (1981) Strong Approximations in Probability and Statistics. New York: Academic Press.

de Acosta, A. and Kuelbs, J. (1983) Limit theorems for moving averages of independent random vectors. Z. Wahrscheinlichkeitstheorie Verw. Geb., 64, 67-123.

Déniel, Y. and Dérriennic, Y. (1988) Sur la convergence presque sure, au sens de Cesàro d'ordre $\alpha$, $0<\alpha<1$, de variables aléatoires et indépendantes et identiquement distribuées. Probab. Theory Related Fields, 79, 629-636.

Gaposhkin, V.F. (1965) The law of the iterated logarithm for Cesàro's and Abel's methods of summation. Theory Probab. Appl., 10, 411-420.

Heinkel, B. (1990) An infinite-dimensional law of large numbers in Cesàro's sense. J. Theoret. Probab., 3, 533-546.

Kiesel, R. and Stadtmüller, U. (1996) Erdős-Rényi-Shepp Laws and weighted sums of i.i.d. random variables. J. Theoret. Probab., 9, 961-982.

Lai, T.L. (1974a) Summability methods for independent, identically distributed random variables. Proc. Amer. Math. Soc., 45, 253-261.

Lai, T.L. (1974b) Limit theorems for delayed sums. Ann. Probab., 2, 432-440.

Lanzinger, H. (1995) Fast sichere Konvergenz bei gleitenden Mitteln von Zufallsvariablen unterhalb des Erdős-Rényi-Gesetzes. Doctoral thesis, University of Ulm.

Lanzinger, H. (1998) An almost sure limit theorem for moving averages of random variables between the strong law of large numbers and the Erdős-Rényi-law. ESAIM: Probab. Statist., 2, 163-183. 
Li, D., Rao, M.B., Jiang, T. and Wang, X. (1995) Complete convergence and almost sure convergence of weighted sums of random variables. J. Theoret. Probab., 8, 49-76.

Martikainen, A. (1982) The strong law for ruled sums. Vestnik Leningrad Univ. Math., 10, 63-74.

Petrov, V.V. (1975) Sums of Independent Random Variables. New York: Springer-Verlag.

Stadtmüller, U. (1984) A note on the law of iterated logarithm for weighted sums of random variables. Ann. Probab., 12, 35-44.

Stadtmüller, U. (1995) On a family of summability methods and one-sided Tauberian conditions. J. Math. Anal. Appl., 196, 99-119.

Received July 1997 and revised April 1998 